\title{
A RAY TRACING METHOD TO SIMULATE THE INFRARED HEATING OF SEMI-TRANSPARENT THERMOPLASTICS
}

\author{
M. Bordival ${ }^{1,2^{*}}$, F.M. Schmidt ${ }^{1,2}$, Y. Le Maoult ${ }^{1,2}$, B. Cosson $^{3}$, B. Plantamura ${ }^{4}$ \\ ${ }^{1}$ Université de Toulouse ; INSA, UPS, Mines Albi, ISAE ; ICA (Institut \\ Clément Ader) ; F-81013 Albi Cedex 09, France - bordival@ mines-albi.fr \\ ${ }^{2}$ Ecole Mines Albi ; Campus Jarlard, F-81013 Albi, France \\ ${ }^{3}$ Ecole des Mines de Douai - BP 10838, 59508 Douai Cedex, France - cosson@ensm-douai.fr \\ ${ }^{4}$ SIDEL - BP204, 76053 Le Havre Cedex, France - Bernard.Plantamura@ sidel.com
}

\begin{abstract}
This paper focuses on RAYHEAT, a software based on the ray tracing method, developed in order to simulate the InfraRed (IR) heating of semi-transparent polymers. In this study, RAYHEAT is used to simulate the IR heating step of a PET preform for the Stretch-Blow Moulding (SBM) process. The general principle of the method is to discretize, into a set of rays, the radiative heat flux emitted by halogen lamps, then to follow these rays inside the oven while they are not fully absorbed. The ray tracer computes the optical path of each ray - accounting for specular or diffuse reflections, refractions, etc... - from its emission point, and throughout the preform thickness. PET is assumed to behave like a non-scattering cold medium. Thus, the radiative heat flux absorption inside the preform is computed according to the Beer-Lambert law. Finally, the distribution of the radiative source term is calculated in the preform. In a second step, the radiative source term is applied as an input data in the commercial finite element software $\mathrm{ABAQUS}^{\circledR}$, in order to calculate the 3D temperature distribution in the preform. The source term is assumed to be time dependent in order to account for the preform movement throughout the IR oven. This method provides relatively small computation times, while keeping the memory requirements down to a minimum. Numerical results have been compared with temperature measurements performed on an in-lab IR oven. The model simulates suitably the infrared heating stage, and provides accurate predictions of the temperature distribution in the preform. The relative error between the temperature calculated by RAYHEAT, and the measured temperature, is less than $5 \%$.
\end{abstract}

KEYWORDS: Ray Tracing, Heat transfer modelling, Stretch blow moulding, Infrared heating, PET absorption

\section{INTRODUCTION}

A large part of bottles intended to beverage market is manufactured using Stretch-Blow Moulding (SBM) process. SBM involves an InfraRed (IR) heating stage to condition the PET preform to the appropriate temperature distribution (around $100^{\circ} \mathrm{C}$ ). Then, the preform is stretched-blown inside a mould to get the final bottle. The heating conditions, which control the preform temperature distribution, strongly affect the final properties of the bottle (mechanical, optical and barrier properties).

Within the last ten years, significant researches have been carried out on the simulation of the IR heating stage. A recent literature review on this subject has been presented by Bordival et al. [1]. PET behaves like a semi-transparent body over the IR spectral range, resulting in major challenges with regard to radiative heat transfers modelling. Different approaches are presented in the literature in order to compute the radiative absorption through the preform thickness. The most classical is the view factors method $[2,3,4]$. This method consists in calculating the radiation on the preform outside surface, and then to assume that the radiative heat flux is unidirectional through the preform thickness. More recent works investigated the efficiency of the zone method [5] or the ray tracing method [6]. The last one presents the advantage to account for a while variety of optical effects, such as specular reflection or refraction. In addition, ray tracing enables to take into account most of constitutive elements of an IR oven like multiple lamps (various geometries) and reflectors.

In this work, we propose a numerical modelling of the IR heating stage. The temperature distribution of the preform is calculated using a two-step simulation. First, in-lab software, called RAYHEAT, is used in order to compute the absorption of the radiative heat flux inside the preform. Then, the results are applied as an input data in the finite element commercial package ABAQUS $^{\circledR}$. The method is applied in order to simulate the IR heating stage of a rotating preform using the inlab blowing machine set-up. A numerical validation is performed using temperature measurements.

\footnotetext{
* M. Bordival: ICA/ALBI, Campus Jarlard 81000 Albi - France, +33-563493085, Fax: +33-563493242, bordival@ mines-albi.fr
} 


\section{HEAT TRANSFER MODELLING}

\subsection{HEAT BALANCE EQUATION}

The evolution versus time of the preform temperature is governed by the transient heat balance equation:

$$
\rho c_{p} \frac{\partial T}{\partial t}=\nabla \cdot(k \nabla T)-\nabla \cdot q_{r}
$$

where $T=$ temperature, $t=$ time, $\rho=$ density, $c_{p}=$ specific heat, $k=$ thermal conductivity, $q_{r}=$ radiative heat flux density. The inside surface of the preform is assumed to be adiabatic, while the following boundary condition is applied to the outside surface:

$$
-\left.k \frac{\partial T}{\partial n}\right|_{P}=h_{c}\left(T_{P}-T_{\infty}\right)+\varepsilon_{P E T} \sigma\left(T_{P}^{4}-T_{\infty}^{4}\right)
$$

where $h_{c}=$ convection heat transfer coefficient, $\varepsilon_{P E T}=$ PET's mean emissivity, $\sigma=$ Stefan-Boltzman constant, $T_{P}=$ outside surface temperature, $T_{\infty}=$ air temperature inside the oven. The boundary condition given by Equation (2) accounts for two types of heat transfer. The first one is due to the cooling by natural or forced convection if oven is ventilated, the second one to the preform own emission. These heat losses have a critical effect, especially throughout the cooling stage.

\subsection{RADIATIVE SOURCE TERM}

Over the spectral range corresponding to the emission of IR lamps $(0.35-8 \mu \mathrm{m})$, PET behaves like a semitransparent body. This involves that the radiative heat flux is partly absorbed inside the wall thickness of the preform, and cannot be simply applied as a boundary condition. The radiation absorption must be taken into account through the divergence of the radiative heat flux (Equation 1), also called "radiative source term".

The radiative heat flux is required to compute the radiative source term. Per definition, the radiative heat flux density is given by:

$$
q_{r}(s)=\int_{0}^{\infty} \int_{4 \pi} I_{\lambda}(s, \Omega) \Omega d \Omega d \lambda
$$

where $I_{\lambda}=$ spectral intensity at the point $s$, along the direction $\Omega$. The variation of the spectral intensity is governed by the Radiative Transfer Equation (RTC), given for a non-scattering medium by [7]:

$$
\frac{d I_{\lambda}(s, \Omega)}{d s}=\kappa_{\lambda}\left[I_{\lambda}^{\circ}(T(s))-I_{\lambda}(s, \Omega)\right]
$$

where $I_{\lambda}^{\circ}=$ Planck's spectral intensity at the preform temperature $T, d s=$ optical path, $\kappa_{\lambda}=$ PET spectral absorption coefficient. Equation (4) has got an exact solution, given by [8]:

$$
I_{\lambda}(s, \Omega)=\overbrace{I_{\lambda}(0, \Omega) e^{-\kappa_{\lambda} s}}^{1}+\overbrace{\int_{s^{\prime}=0}^{s^{\prime}=s} \kappa_{\lambda} I_{\lambda}^{\circ}(T(s)) e^{\kappa_{\lambda}\left(s^{\prime}-s\right)} d s^{\prime}}^{2}
$$

In Equation (5), the first term of the right hand side member represents the fraction of the incident intensity (emitted by the IR oven) which is absorbed by PET. The second term represents the fraction of the intensity, emitted by PET, which is absorbed by PET (due to the own emission).

Throughout the heating stage, the preform temperature (less than $400 \mathrm{~K}$ at the end of heating) is very low compared to the IR heater temperature (typically around $2400 \mathrm{~K})$. In this case, the second term of Equation (5) can be neglected. This assumption leads to:

$$
I_{\lambda}(s, \Omega)=I_{\lambda}(0, \Omega) e^{-\kappa_{\lambda} s}
$$

Equation (6) is called Beer-Lambert law [7]. Then, by assuming that lamps emission is Lambertian, the radiative source term is given by [9]:

$$
\nabla \cdot q_{r}(s)=-\int_{0}^{\infty} \kappa_{\lambda} M_{\lambda}(0) . e^{-\kappa_{\lambda} s} d \lambda
$$

where $M_{\lambda}(0)=$ incident spectral emissive power. The radiative source term distribution is assumed to be temperature-independent. Therefore, it can be computed and then applied as an input data in the finite element software ABAQUS ${ }^{\circledR}$ in order to solve the heat balance equation.

\subsection{RAY TRACING METHOD}

Ray tracing is widely acknowledged as one of the most accurate method used to simulate radiative heat transfers in semi-transparent media. The general principle of the method is to discretize, into a set of rays, the radiative heat flux emitted by halogen lamps. Then, each ray is followed inside the oven, from its emission point and throughout the preform thickness. The ray tracer computes the optical path of each ray, accounting for specular or diffuse reflections, refractions, etc...

In our ray tracing software, assumptions are made for the different optical properties of lamps, reflectors and preforms. Those assumptions are referenced in table 1 for the following properties: emission, absorption, reflection and refraction. Reflection and refraction are averaged in order to reduce the number of ray stored in each calculation. In fact, for one ray that comes from the lamp and contained all the spectral information, if spectral reflection (or refraction) is computed, one ray by spectral band (an infinity for an exact model) has to be created for each air-PET interface crossing.

Table 1: Assumptions on optical properties

\begin{tabular}{lcccc}
\hline Lamps & $\begin{array}{c}\text { Emission } \\
\text { isotropic }\end{array}$ & Absorption & Reflection & Refraction \\
\hline PET & Averaged & Spectral & $\begin{array}{c}\text { Specular and } \\
\text { averaged }\end{array}$ & averaged \\
\hline $\begin{array}{l}\text { Ceramic } \\
\text { reflector }\end{array}$ & none & opaque & $\begin{array}{c}\text { diffuse and } \\
\text { averaged }\end{array}$ & none \\
\hline $\begin{array}{l}\text { Metallic } \\
\text { reflector }\end{array}$ & none & opaque & $\begin{array}{c}\text { Specular and } \\
\text { averaged }\end{array}$ & None \\
\hline
\end{tabular}

Only the lamp filament is taken into account. Filaments are modelled by equivalent cylinders, the spiral form is 
neglected. Tungsten filaments are assumed to be Lambertian grey bodies. This assumption provides the definition of ray direction vectors (Figure 1) for rays coming from the filament.

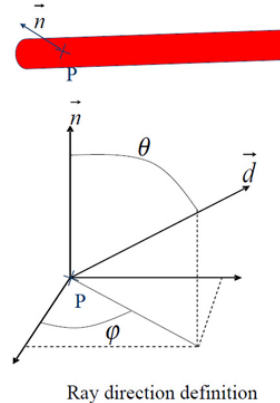

(a)

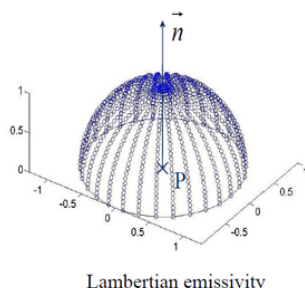

(b)
Figure 1: Ray definition for ray tracing

The direction vector is defined by two parameters: $\theta$ and $\varphi$, respectively defined in the ranges $[0, \pi / 2]$ and $[0,2 \pi]$. Notations are illustrated on Figure 1. The computation of $\theta$ and $\varphi$ has a strong effect on the ray tracing accuracy. Determinist discretization of the emission space could lead to errors due to the ray effect [10]. To avoid that, we have chosen to compute $\theta$ and $\varphi$ according to stochastic variables:

$$
\theta=\arcsin \left(\sqrt{R_{1}}\right) \quad ; \quad \varphi=2 \pi R_{2}
$$

where $R_{1}$ and $R_{2}$ are independent uniform stochastic variables in the range $[0 ; 1]$.

The direction change of a ray that cross a PET-air interface is given by the Snell-Descartes law:

$$
n_{P E T} \sin \theta_{P E T}=n_{\text {air }} \sin \theta_{\text {air }}
$$

where $n_{\text {air }}(=1)$ is the refractive index of air. Notations are illustrated on Figure 2.

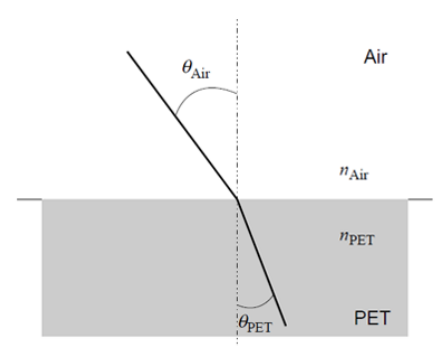

Figure 2: Ray refraction at air-PET interface crossing

\section{NUMERICAL VALIDATION}

\subsection{EXPERIMENTAL DEVICE}

In order to validate numerical simulations, IR heating trials have been performed on an in-lab IR oven. The oven is composed of five halogen lamps $(2 \mathrm{~kW}$ nominal power), with ceramic and back aluminium reflectors (see Figure 3). Percentages of nominal power of each lamp are reported in Table 2 . After $25 \mathrm{~s}$ heating, the preform is cooled down by natural convection during $10 \mathrm{~s}$. No ventilation system is used. The natural convection coefficient was calculated using the empirical correlation of Churchill and Chu [11]. Its value was estimated to be $7.5 \mathrm{~W} \cdot \mathrm{m}^{-2} \cdot \mathrm{K}^{-1}$.

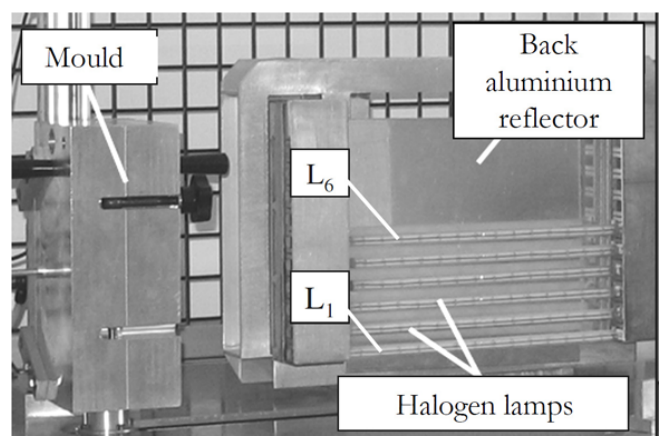

Figure 3: In-lab infrared oven

Table 2: Lamps setting for IR heating trials

\begin{tabular}{cccccc}
\hline & $\mathrm{L}_{1}$ & $\mathrm{~L}_{2}$ & $\mathrm{~L}_{3}$ & $\mathrm{~L}_{4}$ & $\mathrm{~L}_{5}$ \\
\hline Power $(\%)$ & 100 & 100 & 20 & 5 & 60
\end{tabular}

The preform used is $27 \mathrm{~g}$ weight, $3.85 \mathrm{~mm}$ thickness. Its rotational speed is equal to $1.1 \mathrm{rev} / \mathrm{s}$. The reference of polymer is PET INVISTA 1101 (grade VI $=0.8$ ).

Temperature measurements have been performed using an AGEMA 880 LW IR camera, functioning within the long wave spectral range $8-12 \mu \mathrm{m}$. Over this spectral band, PET behaves like an opaque body [12]. Thus, the camera measures a surface temperature.

\subsection{PET THERMAL/RADIATIVE PROPERTIES}

PET radiative properties were measured according to the protocols defined by Monteix et al. [12]. Measurements were performed on PET INVISTA 1101 samples using a Perkin Elmer 950 spectrometer over the range 0.25-2.5 $\mu \mathrm{m}$, and a Perkin Elmer FTIR spectrometer over the range $2.5-25 \mu \mathrm{m}$. PET thermal properties are assumed to be temperature-dependent, which is particularly true for the heat capacity that sharply increases above the glass transition temperature.

\subsection{NUMERICAL PARAMETERS}

The preform is meshed into 5220 rectangular linear elements (6000 nodes), with ten nodes in the thickness direction. For each lamp, 1.5 million rays are followed, for a total of 7.5 millions rays for the entire oven. The computational time of the source term is equal to $90 \mathrm{~min}$ CPU (T9500 2.6 GHz 3Go RAM).

Temperature calculation was performed using ABAQUS $^{\circledR}$ standard 6.7, with a fixed time increment equal to $0.25 \mathrm{~s}$. The computation time is equal to $12 \mathrm{~min}$ CPU.

\subsection{RESULTS AND DISCUSSION}

Figure 4 illustrates the radiative source term distribution versus time. We can observe that the source term has been defined as a function of time in order to account for 
the preform rotation. Figure 5 illustrates the external temperature distribution along the preform height, at the end of the cooling step. We can observe a good agreement between simulations and measurements, since the mean relative error is equal to $1.7 \%$. Figure 6 illustrates the variation of external temperature versus time on a single point, located at $40 \mathrm{~mm}$ from the neck of the preform (middle-height). The agreement is fair, especially during the cooling stage, which indicates the convection heat transfer coefficient is well estimated.

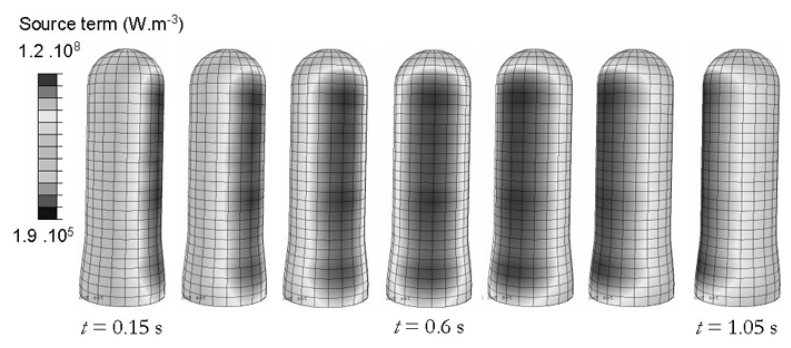

Figure 4: Source term distribution versus time

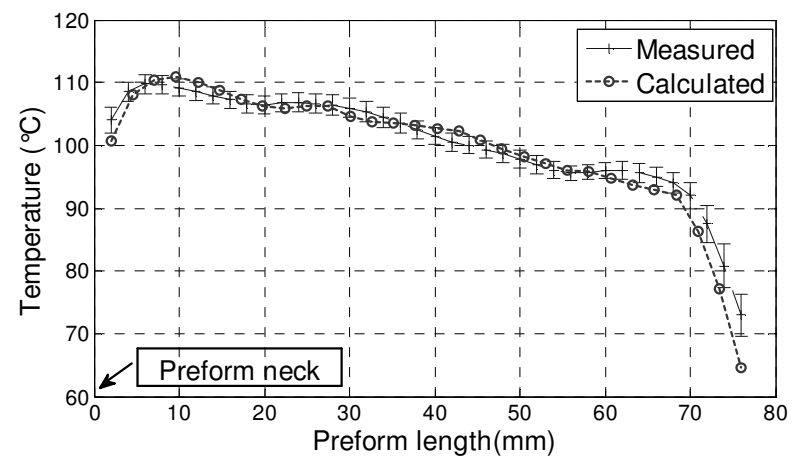

Figure 5: Temperature profile along the preform height

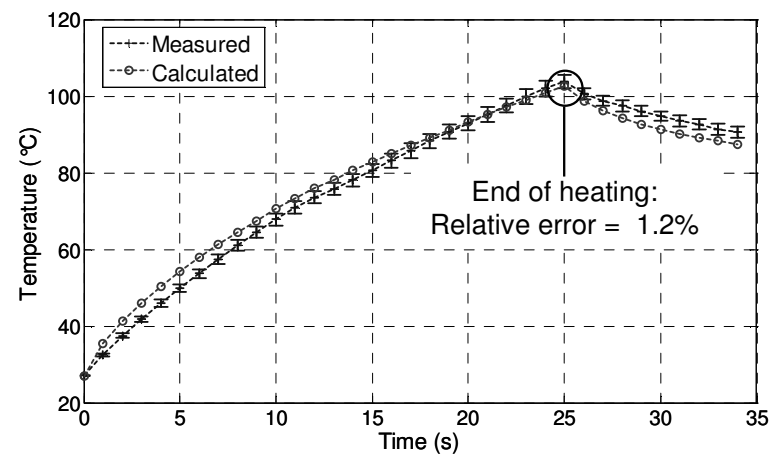

Figure 6: External temperature versus time (mid-height)

\section{CONCLUSIONS}

We have proposed in this work a modelling of the IR heating stage for the SBM process. The ray tracing software RAYHEAT provides accurate predictions of the $3 \mathrm{D}$ radiative source term distribution in the preform. The coupling between RAYHEAT and ABAQUS ${ }^{\circledR}$ allows the computation of the preform temperature, accounting for the preform movement throughout the oven. A numerical validation has shown the efficiency of the method, since the relative error between simulations and experiments is less than $2 \%$. Future work will aim to further improve the ray tracer in order to decrease the computation times and enlarge the validation on larger oven of industrial blow moulding machine.

\section{ACKNOWLEDGEMENT}

Special thanks to SIDEL (http://www.sidel.com) for their collaboration, inputs in some application range of these works, and for their supply of material specifications and preforms.

\section{REFERENCES}

[1] Bordival M., Schmidt F.M., Le Maoult Y., Velay V.: Optimization of Preform Temperature Distribution for the Stretch-Blow Molding of PET Bottles: Infrared Heating and Blowing Modeling. Polym. Eng. Sci. 49:783-793, 2009.

[2] Monteix S., Schmidt F., Le Maoult Y., Yedder R.B., Diraddo R.W., Laroche D.: Experimental study and numerical simulation of preform sheet exposed to infrared radiative heating. J. Mater. Proc. Tech., 119: 90-97, 2001.

[3] Huang H.-X., Deng Y.-H., Huang Y.-F.: Temperature profiles within reheated preform in stretch blow molding. ANTEC'05 (Boston, Massachusetts), May 2005.

[4] Yousefi A., Diraddo R., Bendada A.: Simulation of the mobile preform reheat in injection stretch blow molding process, In PPS-17, The Polymer Processing Society, p. 134-146, 2001.

[5] Michaeli W., Papst W.: FE-Analysis of the TwoStep Stretch Blow Molding Process. In SPE ANTEC Technical Papers 30, Chicago, USA, 2004.

[6] Champin C., Bellet M., Schmidt F.M., Agassant J.F., Le Maoult Y.: 3D Finite Element Modeling of the Blow Molding Process. In 8th ESAFORM Conference on Material Forming, p. 905, 2005.

[7] Modest M.F.: Radiative heat Transfer. McGraw-Hill Inc, 1993.

[8] Viskanta R., Anderson E.E.: Heat Transfer in Semitransparent Solids. Advances in Heat Transfer, 11:317-441, 1975.

[9] Erchiqui F., Hamani I., Charette A.: Modélisation par éléments finis du chauffage infrarouge des membranes thermoplastiques semi-transparentes. International Journal of Thermal Sciences 48:73-84, 2009.

[10]Li B.-W., Tao W.-Q., Liu R.-X.: Ray effect in ray tracing method for radiative heat transfer. Int. J. Heat Mass Transfer, 40:3419-3426, 1997.

[11] Churchill S.W., Chu H.S.: Correlating Equations for Laminar and Turbulent Free Convection from a Vertical Plate. Int. J. Heat Mass Transfer, 18:13231329, 1975.

[12] Monteix S., Le Maoult Y., Schmidt F.M.: Quantitative infrared thermography applied to blow moulding process : measurement of a heat transfer coefficient. QUIRT Journal, 1(2):133-150, 2004. 